\title{
Modeling of Energy Amplification Recorded within Greater Los Angeles Using Irregular Structure
}

\author{
by Chandan K. Saikia, Douglas S. Dreger, and Donald V. Helmberger
}

\begin{abstract}
We have investigated energy amplification observed within Greater Los Angeles basin by analyzing regional waveforms recorded from several Nevada Test Site (NTS) nuclear explosions. Although the stations are located nearly at the same azimuth (distances ranging from 350 to $400 \mathrm{~km}$ ), the seismograms recorded in Compton (the central part of the basin), Long Beach (the southern edge of the basin), and downtown Los Angeles are remarkably different, even for a common explosion. Following the onset of $L_{g}$ waves, the Long Beach sites have recorded surface waves for more than $100 \mathrm{sec}$. From one explosion, the sites within downtown Los Angeles have recorded seismograms with strong 3-sec surface waves. These waves are not observed on the seismograms recorded in the neighboring hard-rock site California Institute of Technology (CIT) station. Thus, they must have been generated by local wave guides. Numerically, we modeled these 3 -sec waves by convolving the CIT seismogram with the response of a sedimentary strata dipping gently (about $6^{\circ}$ ) from CIT toward downtown. We also examined the irregular basin effect by analyzing the variation of cumulative temporal energy across the basin relative to the energy recorded at CIT from the same explosion. Variation up to a factor of 30 was observed. To model the energy variation that is caused by extended surface waves in the Long Beach area, we used numerically simulated site transfer functions (STF) from a NNESSW oriented two-dimensional basin structure extending from Montebello to Palos Verdes that included low-velocity sedimentary material in the uppermost layers. These STFs were convolved with the CIT seismogram recorded from the MAST explosion. To simulate elongated duration of surface waves, we introduced in the upper sedimentary structure some discontinuous microbasin structures of varying size, each microbasin delaying the seismic waves propagating through them. Consequently, the surface-reflected phases through these structures are delayed and reflected into the upper medium by the underlying interfaces. This mechanism helps delayed energy to appear at a later time and result in a longer time duration at sites located at southern edge of the basin.
\end{abstract}

Introduction

Some of the earliest local earthquake seismograms indicate that observations made in basins are amplified relative to hard-rock locations. The relatively strong motions occurring in and around Mexico City during the Michoacan 1985 earthquake is a modern example of this phenomenon. Explanations of these observations are generally attributed to shallow one-dimensional crustal stratigraphy (Borcherdt et al., 1975) or, at longer periods, to trapped waves in irregular basin structure (Vidale and Helmberger, 1988). In the studies related to one-dimensional crustal stratigraphy, the relative groundmotion amplifications are estimated by computing the spectral ratio of the data recorded at a soft-rock site to the data recorded at a hard-rock site, defined as a site transfer function (STF). The STFs are generally determined from weak-motion records and are used to predict future strong motions from large events. In many cases, the amplification of the ground motions is attributed to soil conditions (Idriss and Seed, 1968; Seed et al., 1972) in which lateral homogeneity for the soil column is implicitly assumed. The second approach assumes a distributed shear dislocation embedded in a two-dimensional structure and computes the complete wave field. This approach was used to explain the long-period ( 2 to $10 \mathrm{sec}$ ) waveforms recorded during the 1971 San Fernando, California, earthquake in the neighboring San 
Fernando Valley region (Vidale and Helmberger, 1988). The lateral heterogeneity of surface layers can also play important role in the long duration observed on many seismograms as is the case during the destructive Michoacan, Mexico, earthquake of 1985 (Kawase and Aki, 1989). However, the structure affecting the short wavelength seismic waves is difficult to ascertain, especially in the Greater Los Angeles basin with its many ridges and topographic irregularities. Such features may have caused much of the local variation in seismic intensity observed at various sites in and around the basin during the 1 October 1987 Whittier Narrows earthquake (Kawase and Aki, 1990).

Figure 1a shows the geographic location of the Greater Los Angeles region along with the structural lineaments of the major faults and the basement contours presented in Yerkes et al. (1965). The northern edge of the Los Angeles basin starts near the California Institute of Technology (CIT) and slopes to an approximate depth of about $30,000 \mathrm{ft}$, or $6 \mathrm{~km}$, in the central part of the basin. Geologically, the basin is bounded on the north by the Santa Monica mountains and the Elysian, Ropetto, and Puente

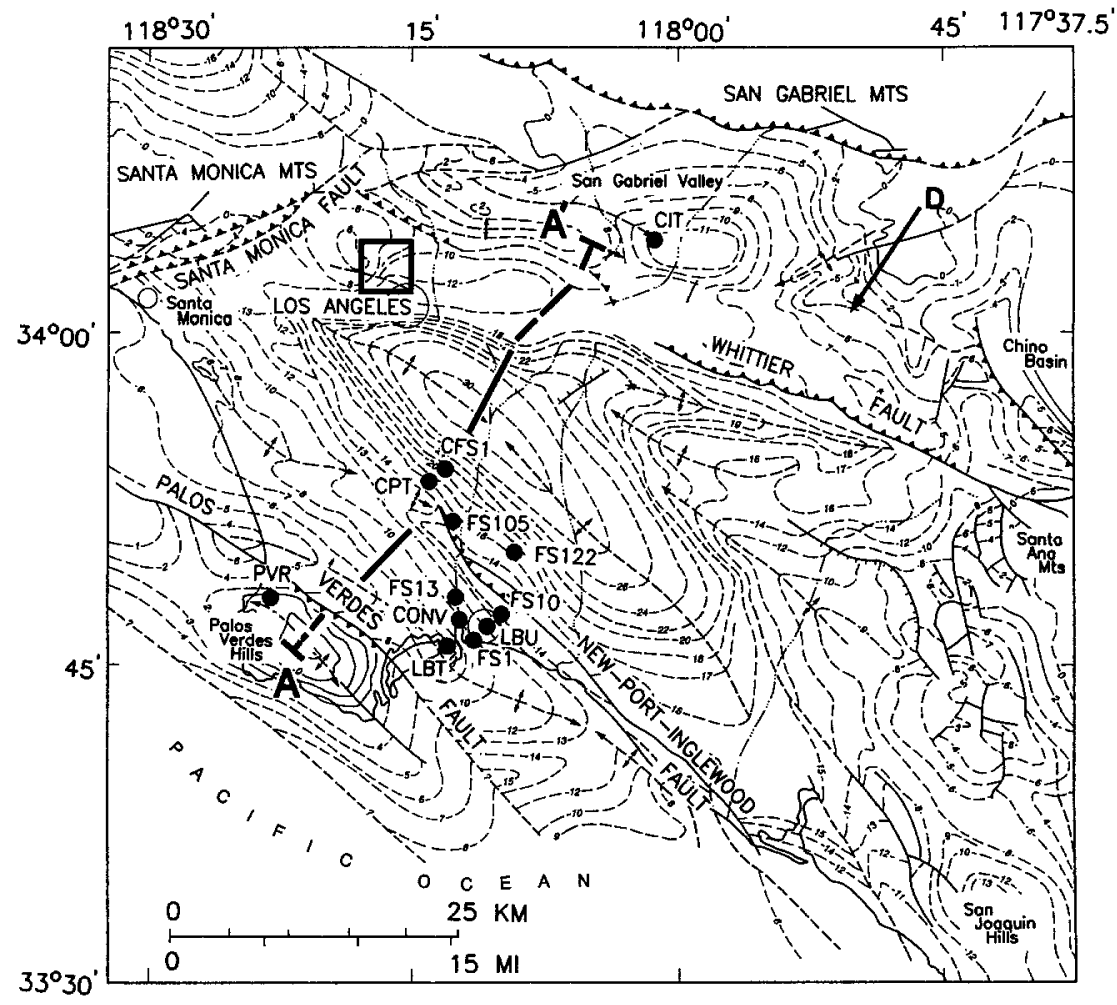

(a)

A HILLS

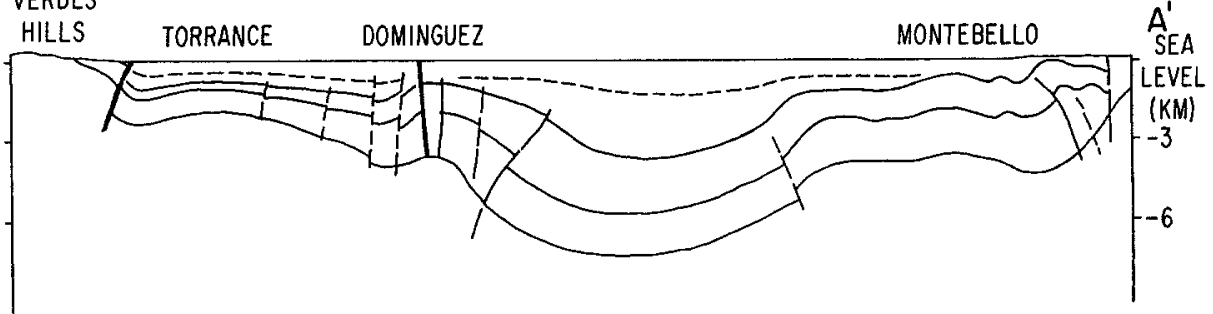

(b)

Figure 1. (a) A map showing the structural lineaments and depths to the basement rock of the Los Angeles basin (modified from Yerkes et al., 1965). The contours are presented in feet in an interval of $1000 \mathrm{ft}$. The stations used in this study are shown by solid circles. The line from $\mathrm{AA}^{\prime}$ shows the orientation of a geological cross section shown in (b). The arrow marked by D shows the direction from Neveda Test Site (NTS) to the Los Angeles basin. (b) Display of a two-dimensional geological cross section $\mathrm{AA}^{\prime}$ shown from Montebello to Palos Verdes. This model is used to investigate the response of irregular basin structure. 
Hills, and by the Santa Ana mountains and San Joaquin Hills on the east and southeast. The south or seaward sloping lowland surface near the northeast margin is interrupted by the Coyote Hills and to the south by a line of elongated hills and mesas extending from the Newport Bay northwest to Beverly Hills. The basin is bounded at the southwest extremity by the Palos Verdes peninsula.

Figure $1 b$ shows a two-dimensional vertical cross section of the Los Angeles basin for a geological profile $\mathrm{AA}^{\prime}$ extending from Palos Verdes at the southwestern edge of the basin to Montebello through the deepest part (after Davis et al., 1989; Wright, 1987). The central part of the basin is interpreted to be a late Miocene to Pliocene graben, and the strata are much thicker than on its outer margin. The basement rock is exposed at both ends of this cross section.

From 1975 to 1979 , the U.S. Geological Survey (USGS) operated a temporary network consisting of threecomponent instruments at many locations in and around the Los Angeles basin to record both regional and local seismograms for the basin response analysis. Several nuclear explosions from NTS, located at distances ranging from 350 to $400 \mathrm{~km}$, were recorded. Rogers et al. (1979) studied the basin transfer function by examining the peak ground velocity (PGV) and spectral ratios of these recorded seismograms at sites of alluvium versus hard rock. Based on the hard-rock ground motions recorded at CIT and Palos Verdes (PVR), their study suggested that the PGV recorded at Long Beach Region can have spectral ratios as large as 11 in the period range from 0.2 to 6 sec. These seismograms exhibit variation not only in the PGV and spectral ratios, but also indicate a great deal of variability in the time domain features. For example, the duration of the surface waves is significantly longer at Long Beach sites compared with the neighboring CIT from the same explosion. In the present study, we examine the possible causes of observed wave-field variation at sites located in close proximity by numerically modeling the observed time histories and the observed energy amplification. We have chosen three sites, the areas in the vicinity of Long Beach, downtown Los Angeles (indicated by the box in Fig. 1a) and Compton, simply because the sites have been heavily urbanized. The basin response is expected to be crucial at these locations for the seismic safety of the existing and future developments during a large earthquake, and there have been ample recordings to estimate the basin response. Figure 1a shows that the basement structure is truely a threedimensional structure. In a similar environment, Frankel and Vidale (1992) showed that such a complicated threedimensional structure can produce $S$ - to surface-wave conversion at the edges of the basin and may produce large amplitude and long duration of ground motion in the basin compared with the surrounding rocks.

We used seismograms from the NTS explosions so that the wave fields arrive at the recording sites almost at the same azimuthal angle. Because the CIT station is located at the northern edge of the basin, it is closest to the source and represents the wave field prior to interacting with the basin. Furthermore, because the length of the array is $<10 \%$ of the total distance to the source region, it is reasonable to assume that this variation in wave field across the basin is directly related to the propagational effects within the basin. Thus, our approach is to use the site transfer function method and transfer the CIT motions to locations in the basin by convolution with two-dimensional numerical operators.

We will first review the data set. This will be followed by temporal energy consideration indicating the large variations at different locations in the basin. The wave-field modeling at the downtown array is discussed next because it is the simplest. This followed by a discussion of the various two-dimensional models of the basin where the effects of the microbasins are introduced.

\section{Data and Characteristics}

First, we show evidence that two regions, Compton and Long Beach, have consistently different $P_{g}$ and $L_{g}$ waves. We analyzed three-component analog seismograms recorded on the instruments installed at stations PVR, 800WS, 611, 464, 420, 445, CIT, FS1, CFS1, CPT, FS122, FS10, FS105, LBT, LBU, and CONV from five NTS explosions: BILLET, CAMEMBERT, MAST, POOL, and STRAIT (Table 1). BILLET and STRAIT are Yucca Flat explosions and the other three are Pahute explosions. All the explosions were below the water table. Figure 1a shows the locations of the stations on a map of major structural features and contours of depth to the basement rock in the Los Angeles basin. Stations 800WS, $611,464,420$, and 445 are located within downtown Los Angeles (LA). The seismograms were recorded by an L-7 seismograph system, which has a flat velocity response for the ground motion in the range from 0.03 to $10 \mathrm{sec}$. The seismograms were digitized and corrected to ground velocity by Rogers et al. (1980). They were filtered using 0.1 - and $10-\mathrm{Hz}$ corners.

The direction D marked by an arrow in Figure 1a shows the direction for the rays approaching the Los Angeles basin from NTS, which is roughly at a distance of $380 \mathrm{~km}$. The basin is located at an azimuth of about $210^{\circ}$ from the Pahute explosions and $203^{\circ}$ from the Yucca explosions. The individual phases recorded on seismograms at stations in Compton and Long Beach show remarkable differences (Fig. 2). The figure shows the threecomponent seismograms recorded at FS122, CPT, CFS1, and FS105 stations in Compton from the CAMEMBERT explosion $\left(M_{\mathrm{b}}=6.2\right)$ and at FS13, FS10, CONV, and FS1 stations in Long Beach from the MAST explosion $\left(M_{\mathrm{b}}=6.1\right)$. The seismograms of CIT are shown for reference. Note that the vertical components at CIT are similar for the two events as expected at a hard-rock site, 
Table 1

The Locations of the Explosions Used in this Study Taken from Rogers et al. (1980)

\begin{tabular}{lccccc}
\hline \multicolumn{1}{c}{ Name } & Date & Time (GMT) & Latitude, ${ }^{\circ} \mathrm{N}$ & Longitude, ${ }^{\circ} \mathrm{W}$ & $M_{\mathrm{b}}$ \\
\hline Mast & 19 June 1975 & $13 \mathrm{hr} 00 \mathrm{~min} \mathrm{0.09} \mathrm{sec}$ & 37.351 & 116.320 & 6.1 \\
Camembert & 26 June 1975 & $12 \mathrm{hr} 30 \mathrm{~min} 0.16 \mathrm{sec}$ & 37.279 & 116.369 & 6.2 \\
Pool & 17 Mar 1976 & $14 \mathrm{hr} 15 \mathrm{~min} 0.09 \mathrm{sec}$ & 37.256 & 116.329 & 6.1 \\
Strait & 17 Mar 1976 & $14 \mathrm{hr} 45 \mathrm{~min} 0.09 \mathrm{sec}$ & 37.107 & 116.053 & 5.8 \\
Billet & 27 July 1976 & 20 hr 30 min 0.07 sec & 37.075 & 116.044 & 5.3 \\
\hline
\end{tabular}

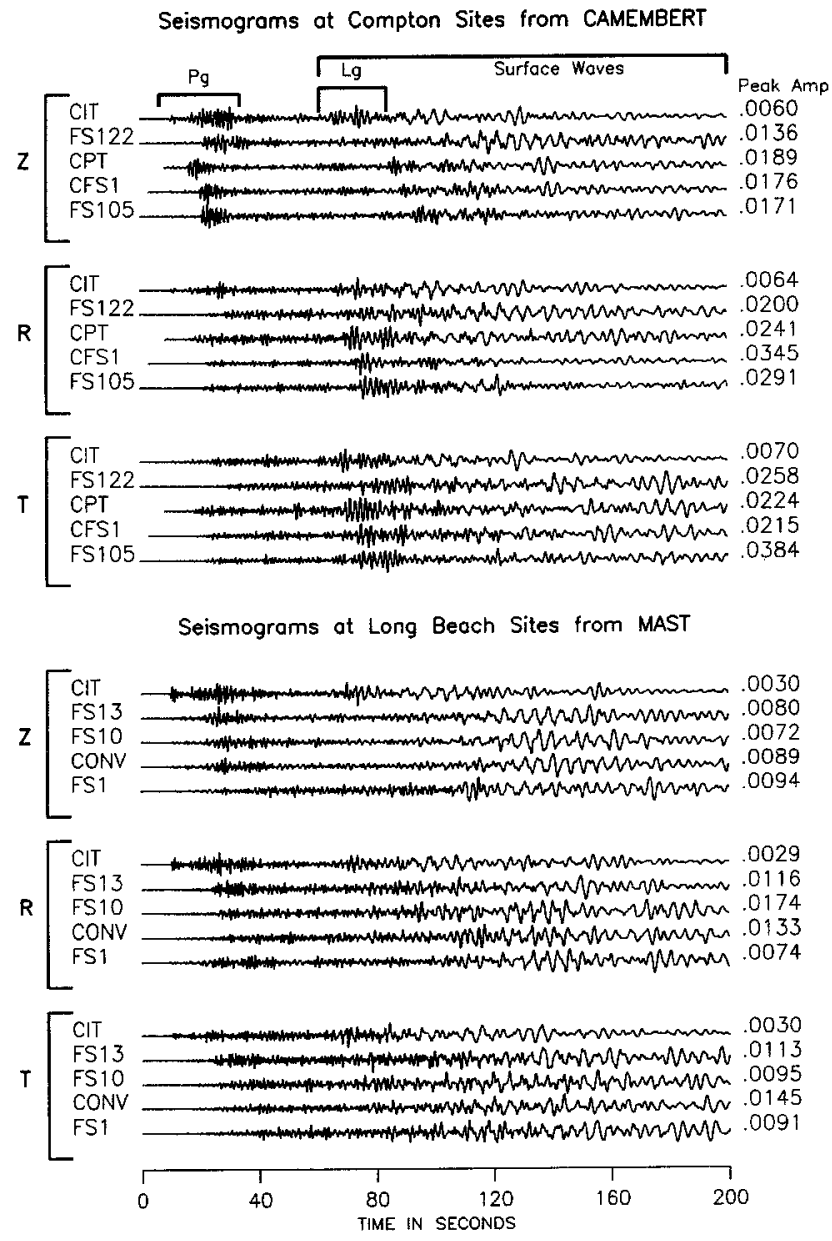

Figure 2. Three-component seismograms at different stations of the Compton and Long Beach area. The Compton stations recorded the CAMEMBERT explosion, and the Long Beach stations recorded the MAST explosion. (The seismograms are aligned so that the $P_{n}$ waves line up.)

but the seismograms recorded at Compton and Long Beach are quite different.

The seismograms show characteristic features at both sites for the $P_{g}$ waves that are the crustal reverberation phases. The interesting aspects of these high-frequency $P_{g}$ waves are the duration and the complexity. The lateral heterogeneity of the basement structure and inclusion of laterally varying crustal material near the surface of a basin structure can significantly affect the interface transmission/reflection coefficients and the timing of rays at each receiver. The effect can be the evolution of $P_{g}$ waves differently at different sites. The complexity of the $P_{g}$ wave guide within the source region and most of the propagation path is expected to remain the same for both explosions. At Compton, the $P_{g}$ energy is mostly on the vertical component and the $S V$ energy is on the radial component. This energy partition is easily explainable by a plane-layered crustal structure with $P$ - and $S$-wave velocities decreasing to the surface. Thus, the $P$ wave field becomes strong on the vertical and the $S$ wave field becomes strong on the radial component.

The energy partition at the Long Beach sites is also noticeably different. The $P_{g}$ wave field is recorded on the radial component as strongly as on the vertical component. The $S V$ energy is not as strong on the radial component as is recorded in the Compton sites. At these sites, the waves must be arriving more horizontally than the sites at Compton. An examination of the geologic map presented in Figure 1 supports these general features. That is, most of the Compton stations are located on thicker and softer sediments, whereas the other set of stations are along the complex basin edge.

The surface waves also display regionalized behavior. For example, the Long Beach sites recorded surface waves of much longer duration on all three components compared with the Compton sites. The wave train starts with significant energy at the arrival of the fundamental mode, which on average continues for about $100 \mathrm{sec}$. On the other hand, the seismograms from the Compton sites have strong surface waves only in the $L_{g}$ wave window. However, the horizontal peak amplitudes show that the site amplifications relative to CIT are similar at two sites, between 2.5 and 5.9 (from surface waves after the $L_{g}$ arrival) at Long Beach stations and between 3.1 and 5.5 (from $L_{g}$ waves) at Compton stations.

Figure 3 shows the radial and tangential seismograms recorded at CIT, LBU, LBT, and PVR from two explosions: POOL $\left(M_{\mathrm{b}}=6.1\right)$, a Pahute event, and STRAIT $\left(M_{\mathrm{b}}=5.8\right)$, a Yucca Flat event. More energetic $P_{g}$ and $L_{g}$ waves are recorded from the POOL explosion at stations LBU, LBT, and PVR than from the STRAIT explosion. The seismograms do have long duration for both Rayleigh and Love waves. Although the waveforms of 
these explosions differ in specific detail, the total duration and the characteristics of the waveshapes are similar. An important feature is that both CIT and PVR are hard-rock site stations and the seismograms have similar peak amplitude for each explosion; the amplitudes are small compared with the peak amplitudes recorded at LBT and LBU. The enhanced peak amplitudes at LBT and LBU are due to an increased impedance contrast caused by the soft materials that overlie the hard-rock materials below them. Although the peak amplitudes are comparable to the peak amplitudes at the CIT station, the seismograms at the PVR station show surface waves (except for the radial component for the POOL explosion) longer in duration compared with the surface waves observed at the CIT station. This may be caused by some complex structure beneath and around the PVR station.

Figure 4 shows the locations of the downtown Los Angeles stations. The stations are distributed in the small area near the civic center, which is marked by the open square. These stations were installed temporarily and recorded only the BILLET $\left(M_{\mathrm{b}}=5.3\right)$ explosion. The most important feature of these seismograms at these down-
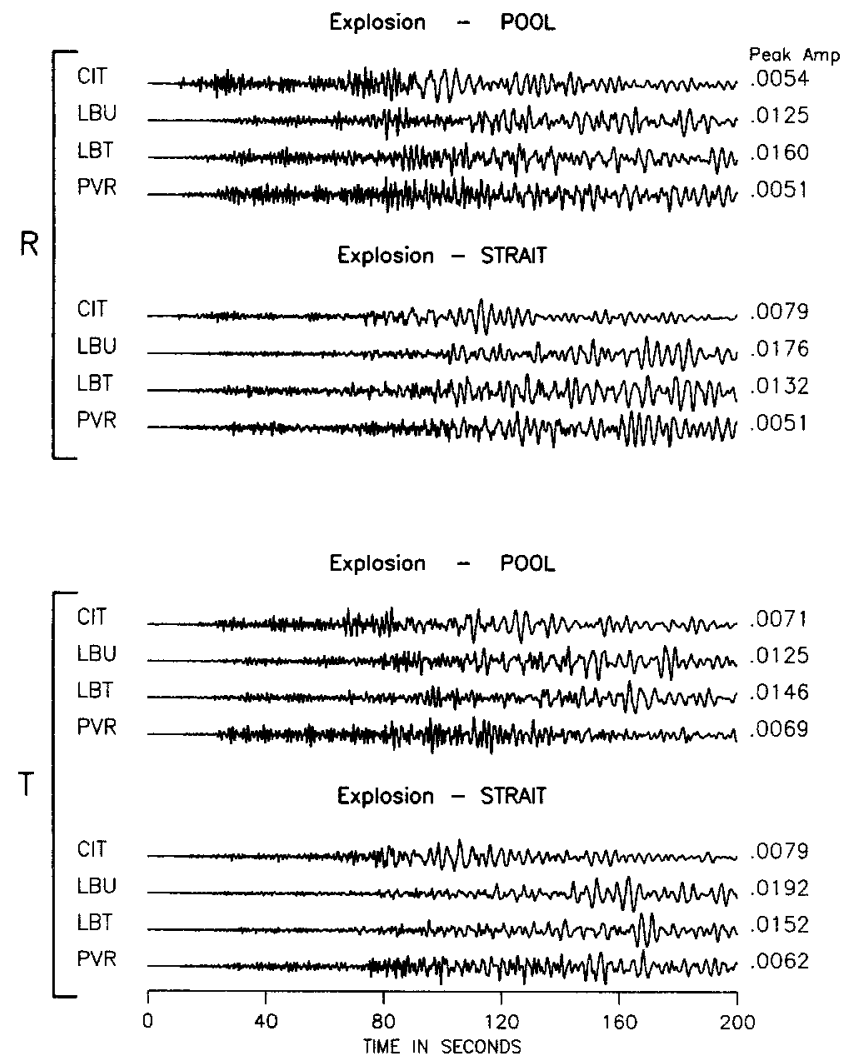

Figure 3. The radial and tangential seismograms recorded at CIT, LBT, LBU, and PVR stations from POOL and STRAIT explosions. Similarities in the CIT seismograms from these two explosions are more marked than at other stations. In this and the remaining figures, the station codes are given on the left, and the peak amplitudes are given on the right. town stations is the strong excitation of Love waves compared with the seismograms recorded at the CIT station. A strong 3-sec period wave is observed on the tangential seismograms of the downtown stations; this is not recorded at the CIT station (Fig. 5; a detailed discussion will be presented in a later section). From the contours

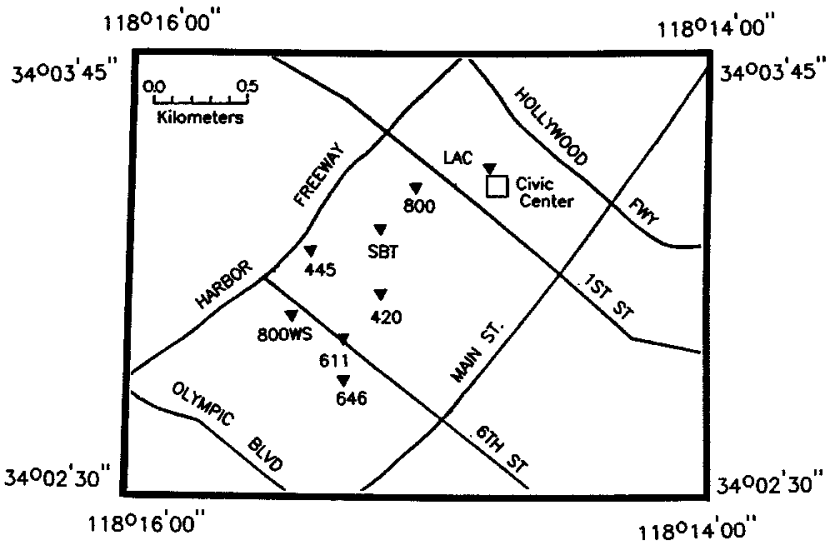

Figure 4. Map showing geographical location of stations in downtown Los Angeles.

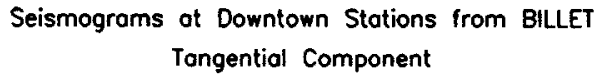

Seismograms of Downtown Stations from BILLET Tongential Component

$\mathrm{ClT}$
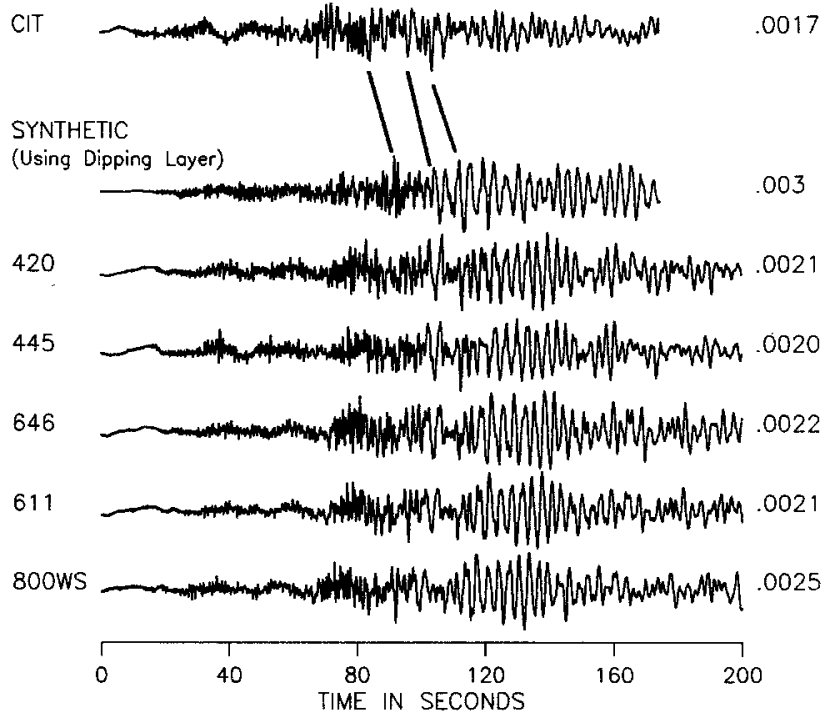

Figure 5. Tangential seismograms are shown at five different stations $[420,445,646,611$, and 800WS (see Fig. 4); all located on soft materials] installed in the downtown Los Angeles area. The second seismogram is a theoretical seismogram modeled using the RSTF method discussed later. Arrows show the correspondence of various phases that are excited using irregular crustal response using California Institute of Technology (CIT; 13 $\mathrm{km}$ from downtown; a hard-rock site) seismogram. The seismograms are recorded from the BILLET explosion. 
of the basement structure shown in Figure 1a, it is reasonable to assume that there exist dipping sedimentary strata from the CIT station toward downtown. These dipping strata can provide wave guides for the regional wave field to be trapped causing the long-period signals to develop.

\section{Temporal Energy Variations at Recording Sites}

A useful measure of strong motions can be obtained by examining an integral energy function $F(t)=$ $\int_{0}^{t}[f(t)]^{2} d t$ as a function of time where $f(t)$ is the ground velocity. This integral energy function, called the temporal energy, has an important property. It can show whether a strong arrival has appeared on a seismogram or a wave type has contributed significantly over a long duration. For illustration, we show the variation of temporal energy at the CIT and CPT stations from the CAMEMBERT explosion in Figure 6. The seismograms are included to illustrate how the arrivals of individual phases have influenced the shape of the energy versus time curve. The windows for regional $P_{g}, L_{g}$, and surface waves are indicated by the dark lines. Energy is normalized by dividing the peak amplitude by the reported body-wave magnitude of each event. Clearly, the energy of every regional phase is amplified at the CPT station relative to the phases at the CIT station. The curve shows an increase in the cumulative energy at the arrival time of each of these phases at the CPT station. The cumulative energy increases monotonically at later times, which is caused by the dominant surface waves trailing at the end of the CPT seismogram.

Because the stations that recorded the seismograms from these NTS explosions lie nearly at the same azimuth, the observed variations in the temporal energy can provide an important clue to distinguish whether the variations are caused by the site specifics or by the propagation effects from the source region. These two effects cannot be separated from each other. Figure 7 shows the variations of the normalized temporal energy versus time from all three-component seismograms of four explosions. For a single event, data from all the stations are plotted in one rectangular panel. The effect of $P_{n}, P_{g}$, $L_{g}$, and surface wave arrivals are included in these curves.

Temporal Energy at CIT and CPT from CAMEMBERT
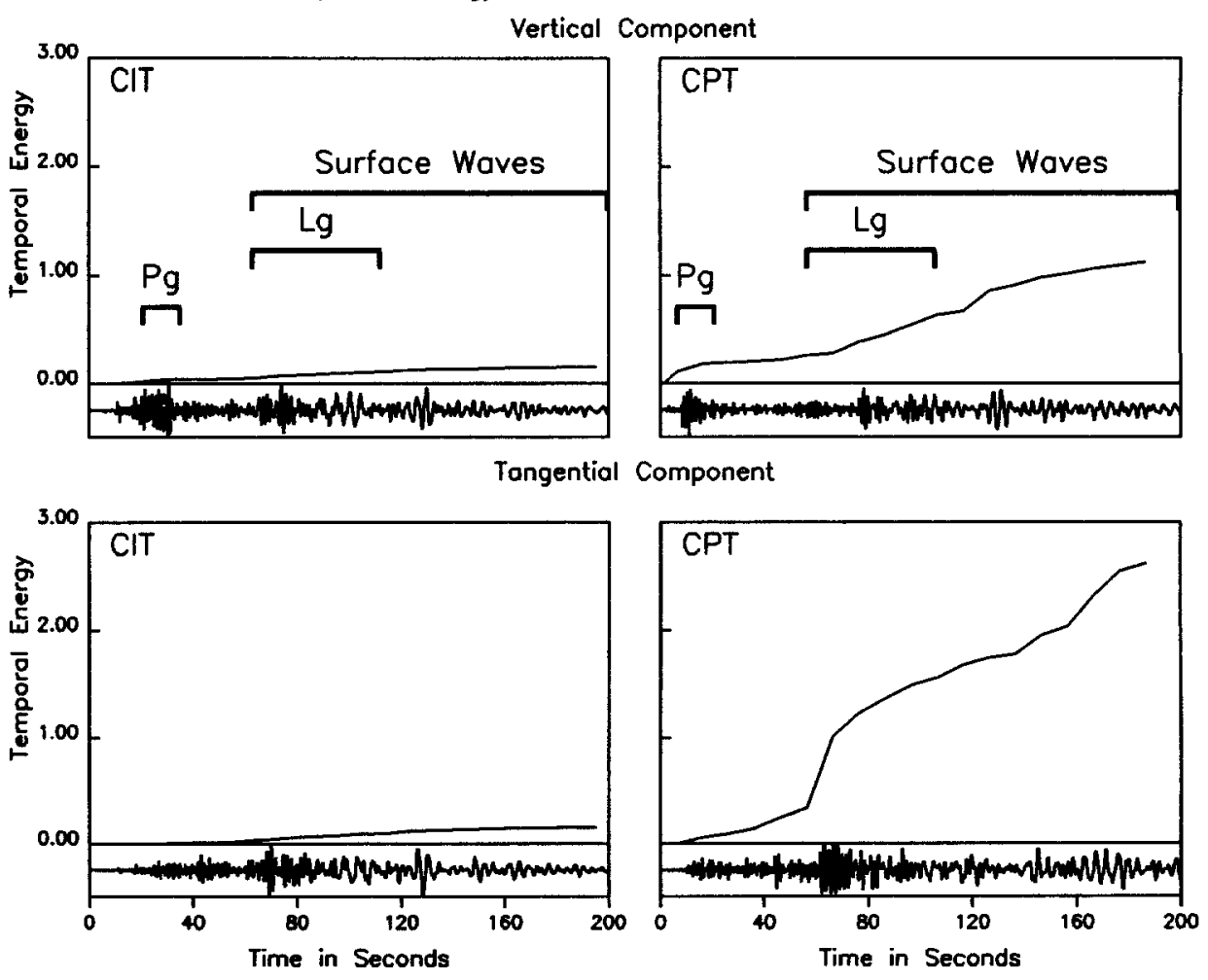

Figure 6. Diagram showing the normalized temporal energy function $F(t)=$ $\int_{0}^{\infty} f(t) d t$ versus $t$ computed for two seismograms recorded at the CPT and CIT stations from the CAMEMBERT explosion. The normalization was done by dividing $F(t)$ by the reported body-wave magnitude of Rogers et al. (1980). The $P_{g}, L_{g}$, and surface wave windows used in this study are shown. The corresponding time domain seismograms are also shown. 
CIT is the first station to interact with the incoming wave field to the basin and its energy maintains a smooth level. The temporal energy at the CIT station stops abruptly at $180 \mathrm{sec}$ for the BILLET explosion, and we do not expect the curve to exhibit additional increase because the significant energy is concentrated mostly in the first 120 sec of the seismograms. The Compton sites that recorded the CAMEMBERT explosion show the highest level of energy fluctuation from station to station. Because all stations lie along one azimuth from the NTS, for a given explosion the source effect should vary negligibly. Therefore, it can be assumed that the above variability in the temporal energy is caused by the specifics of the basin and local site geology rather than by the propagation effects from the source region, the local site geology causing an increase in the amplitude of the waves traveling from a high-velocity medium to a low-velocity medium, and the irregular basin trapping seismic waves within it causing the strength of the later waves to effectively produce a long duration.

TEMPORAL ENERGY VS TIME AT 4 SITES

Explosion: MAST - June 19, 1975
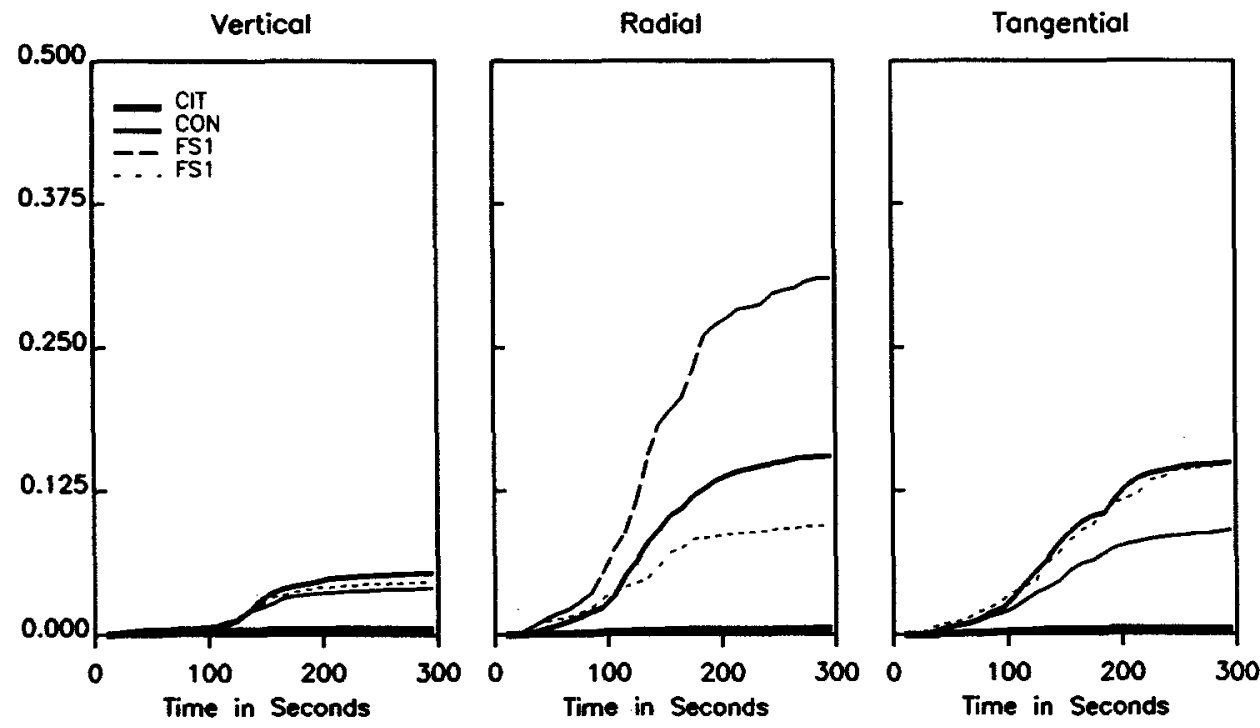

TEMPORAL ENERGY VS TIME AT 4 SITES

Explosion: POOL - March 17, 1976
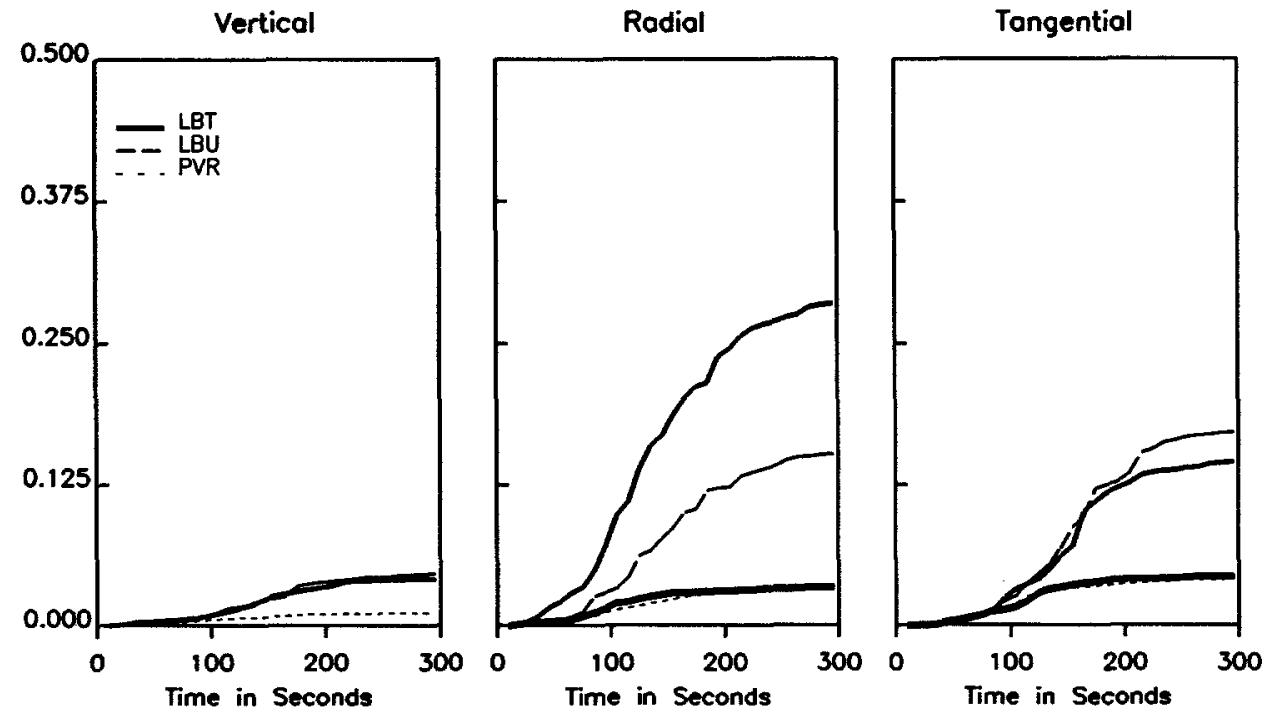

Figure 7. The temporal energy versus time curves for the vertical, radial, and tangential seismograms for four NTS explosions. The solid thick line corresponds to the temporal energy recorded at the CIT station. 
TEMPORAL ENERGY VS TIME AT 5 SITES

Explosion: CAMEMBERT - June 26, 1975
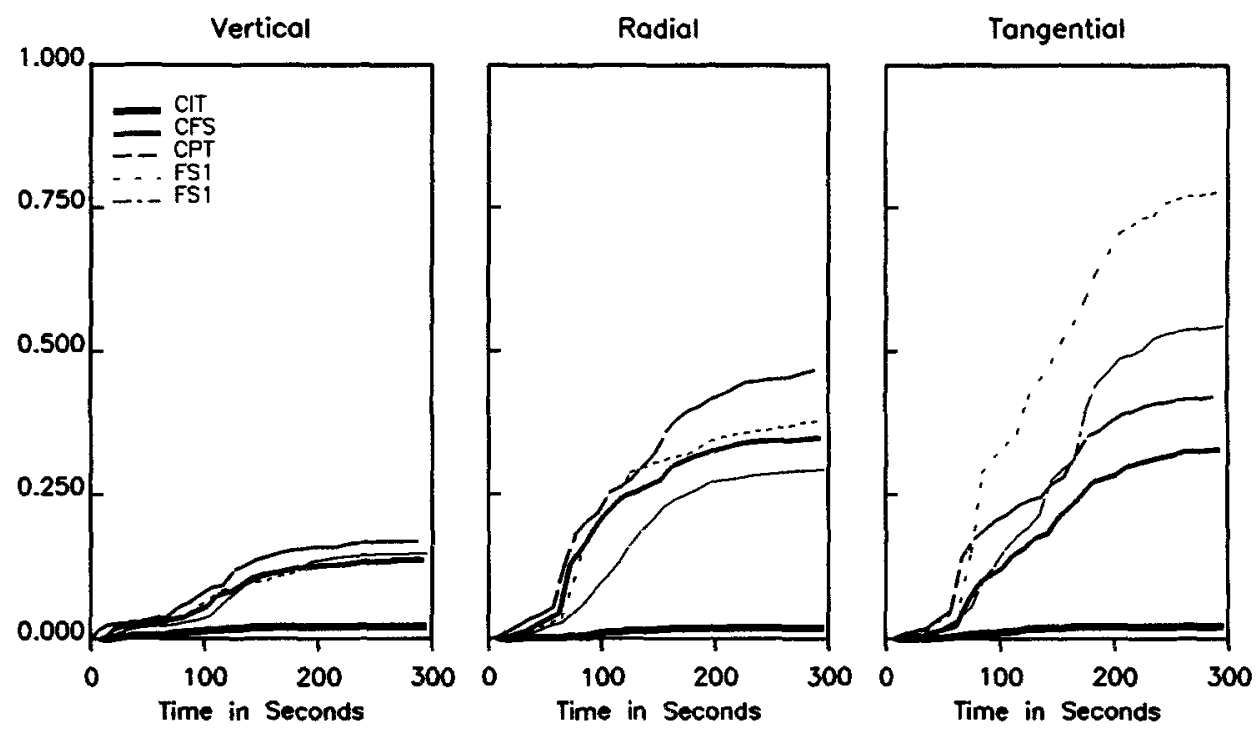

TEMPORAL ENERGY VS TIME AT 5 SITES

Explosion: BILLET - July 27, 1976
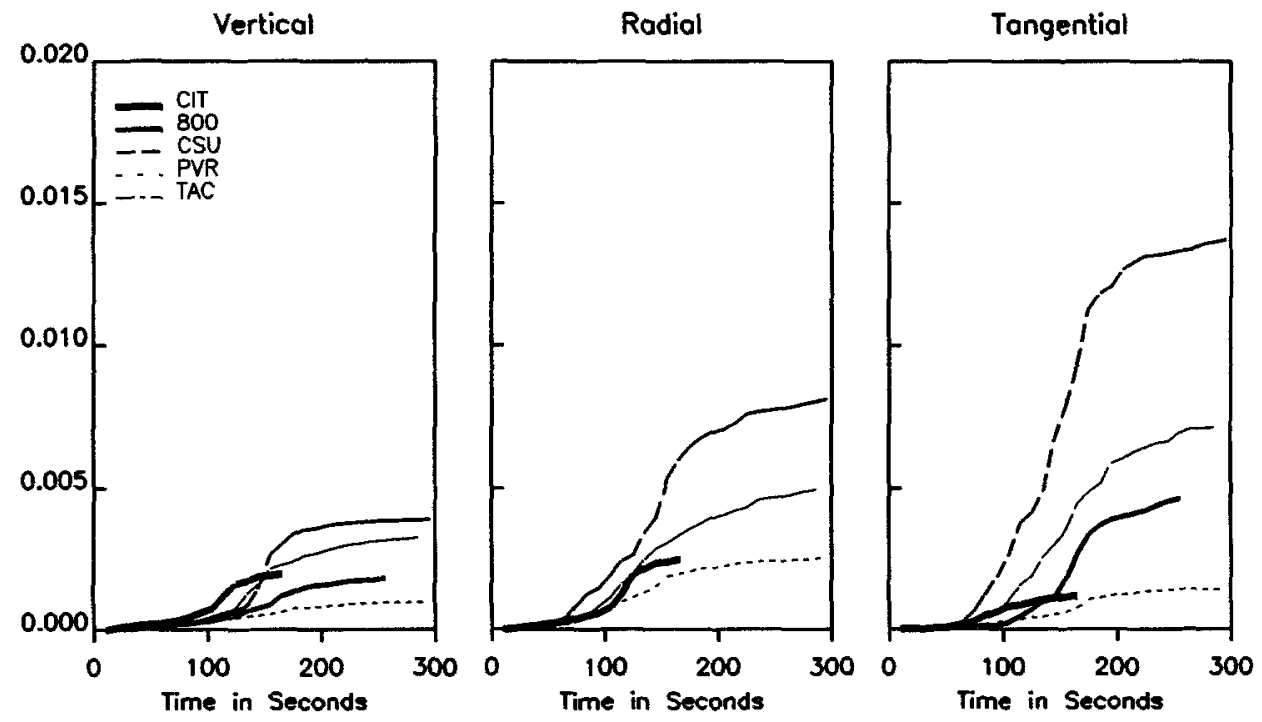

Figure 7. Continued

\section{Modeling of Wave Field at Downtown Los Angeles}

In an earlier section, we stated that the locally dipping sedimentary strata could become a local source for trapping seismic wave fields incident from regional distances. Figure 8 illustrates this phenomenon in terms of ray tracks. The top panel shows the rays leaving an irregular source structure to a receiver at a distance of about $350 \mathrm{~km}$, a range similar to that from the NTS to the Los Angeles basin. At these frequencies, the crustal wave guide is quite complicated, and it is difficult to predict the wave field as it arrives at Pasadena (CIT). However, it probably contains contributions from many different ray paths involving a variety of ray parameters (Saikia and Burdick, 1991). As these rays arrive at the basin structure they will be locally trapped and multi-pathed (Fig. 8, bottom). It is this local phenomena that we will attempt to explain.

In Figure 9 we display an schematic example of how local trapping can produce wave trains with long duration, although it is obviously oversimplified. We fixed the source and receiver at a horizontal offset of $13 \mathrm{~km}$ and assumed a sedimentary structure with a dip of $1.5^{\circ}$, top (a). We choose this geometry to approximate the incoming wave field from the NTS at the edge of the Los 
Angeles basin, essentially in Downtown. The true basement structure dips from about $2 \mathrm{~km}$ at the CIT station to about $2.34 \mathrm{~km}$ at downtown Los Angeles (see Fig. 1a). Figure $9 \mathrm{~b}$ displays the $S H$ step response of the medium for various shear-wave velocities generated with generalized ray theory (Helmberger et al., 1985). Figure $9 \mathrm{c}$ shows the corresponding ray paths that produced the various pulses. The dipping structure allows the internal reflections in the layer to reach critical angle that is responsible for the postcritical angle spikes; see the above reference for details.

Locally dipping structures can explain the extended wave trains, but representing the incoming wave field remains a difficulty. Following the STF approach, one simply convolves the hard-rock site response, for example the response at the CIT station, with a simulation of the flat-layered structure assuming vertical plane waves. In Figure 5, discussed earlier, we displayed the $S H$ responses at the CIT and Downtown stations. Also displayed is the synthetic $S H$ seismogram computed at the locations of downtown Los Angeles stations. The three solid lines show three distinct phases observed at the CIT station and their correspondence at the downtown stations. The synthetic displayed in this figure was produced following this approach. That is, the CIT station observation was convolved with the step response fol-

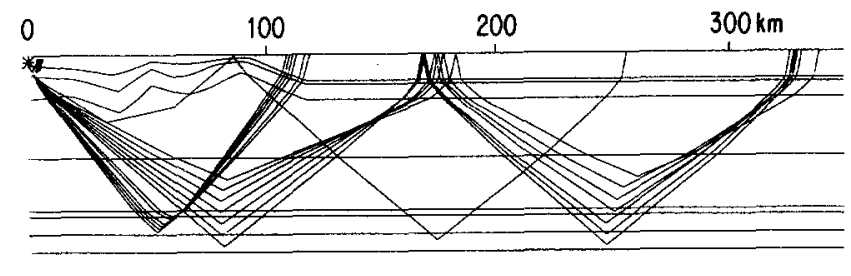

(a)
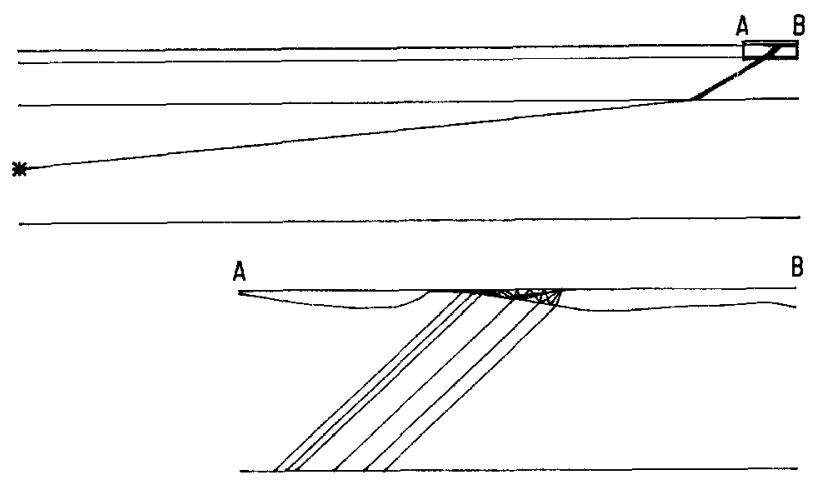

(b)

Figure 8. Diagrams of ray tracks through irregular crustal structures from regional distances. (a) Ray leaves irregular source structure, (b) ray interaction with a receiver having a flat-layered structure, and (c) ray interaction with a receiver having irregular structure beneath it. lowed by a time derivative. The correspondence between the synthetic and observations is quite good, indicating that a dipping structure is, indeed, consistent with the observed durations. The long-period characteristic observed following the correspondence of first phase is also simulated on the synthetic seismogram consistent with data.

However, the amplitude scaling remains a problem, because we need to normalize these point source responses. One simple approach is to correct for geometrical spreading, assuming a flat-layered response. Thus, we first approximate how the CIT station would appear at various positions across the basin with the complex structure removed and the structure approximated by the flat-layered structure beneath the CIT station. We use these amplitudes to correct the complex Green's functions here in Figure 9 for spreading and call these new Green's functions relative site transfer functions (RSTF) to distinguish them from STFs.

\section{Modeling of Wave Field at Long Beach Using Irregular Structure}

The basin structure is more complex than the above example as is reflected in the two-dimensional crustal structure shown in Figure 1b. In this section, we explore two-dimensional crustal structures to explain the observed variation in the temporal energy and waveform characteristics. We used three crustal structures (corresponding to the cross section $\mathrm{AA}^{\prime}$ in Fig. 1) shown schematically in Figure 10 for computing responses using the finite-difference numerical tools (Vidale et al., 1985; Vidale and Helmberger, 1988; Alford et al., 1974; Alterman and Karal, 1968). The $P$-wave $\left(V_{P}\right)$ and $S$-wave $\left(V_{S}\right)$ velocities are in kilometers per second, and the densities are in grams per cubic centimeter. In the lower two models, instead of a continuous-surface sedimentary layer this layer is broken in several discontinuous pockets of low-velocity sedimentary materials represented by the solid triangles. These low-velocity pockets of sedimentary materials delay the reflected wave field at the free surface, which is again reflected back into the upper medium by the basement structure of the basin (see Dreger and Helmberger, 1990). The process continues until it reaches a receiver location and causes the coda waves to develop.

In a similar investigation, Kawase and Aki (1989) simulated long duration of the ground motion observed in Mexico City from the 1985 Michoacan, Mexico, earthquake using a discrete wavenumber boundary element method (DWBEM; Kawase, 1988). They noted that a basin of type 2 in which a soft-surface layer is contained within a deep basin can generate signal duration comparable to the observed duration. Their study was limited to characterizing the basin structure so that the long duration observed in real data could be interpreted. 
Thus, the chosen model was oversimplified. In the present investigation, we explore the effect crustal structure that may be somewhat realistic for the Los Angeles basin. As shown in Figure 10, the soft-layer basin is characterized by several discontinuous small basins and thus is introduced differently from Kawase and Aki (1989). The low-velocity sedimentary basins distributed in this manner produce more complex effects because the basement of the entire basin is also specified underneath these structures. The complexity of the waveforms is entirely dependent on the size of the basins including the attenuation defined by the material properties.

Our objective is to simulate the wave-field recorded at Long Beach from the NTS explosion MAST relative to the wave field at the CIT station using the RSTF approach. The finite-difference $S H$ seismograms between the CIT and Long Beach stations are computed at 16 freesurface receivers for a point source located at a depth of $13.5 \mathrm{~km}$. Of these, the effects observed at stations 12 through 16 should be pertinent to the Long Beach sites. A finite-difference computation is limited by the size and complexity of the problem, and the capability of a com-

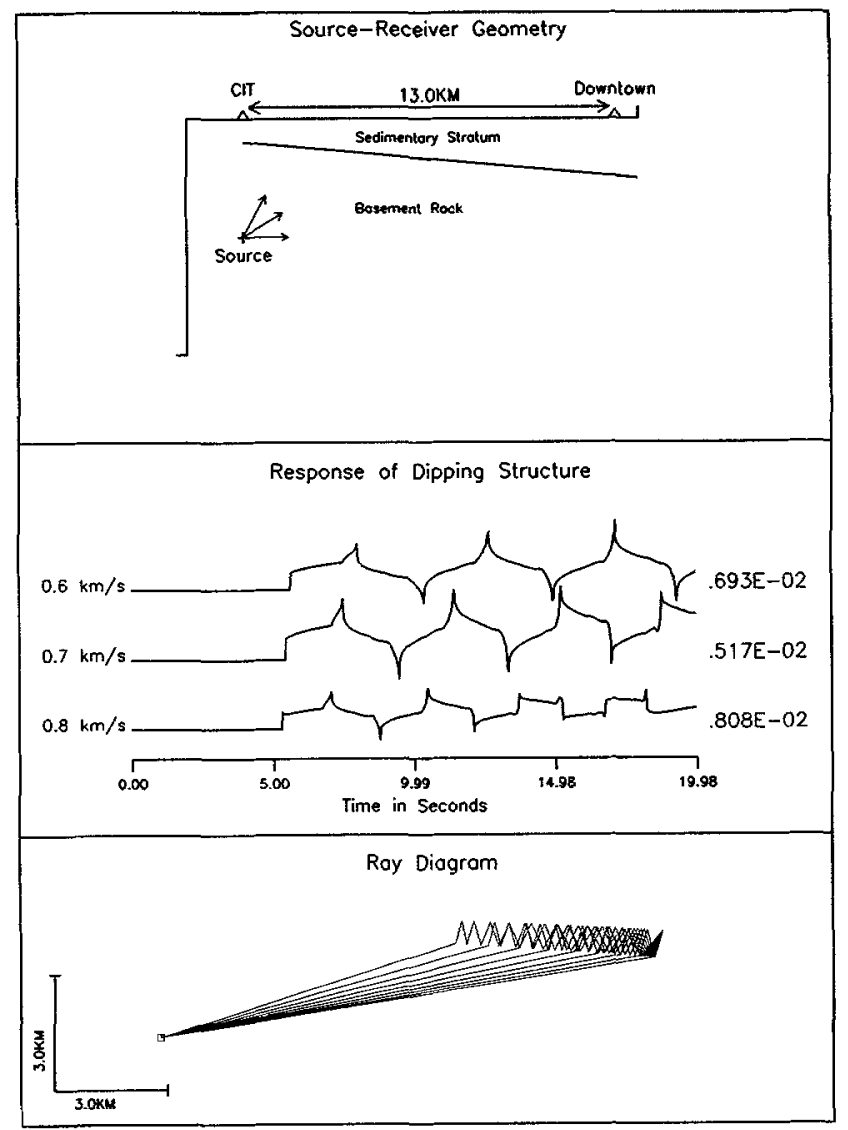

Figure 9. Response of a dipping crustal structure. (a) Source geometry, (b) step response of the dipping crustal structure, corresponding to three shear-wave crustal velocities, and (c) ray tracks used to compute step responses shown in (b). puting machine. Therefore, the algorithms are based on the choice of grid configuration and artificial boundary conditions, finite-difference formulas, and allowable accuracy. A large grid size would introduce a dispersion, delaying higher signal frequencies relative to the lower signal frequencies. Generally, about 10 points per wavelength are needed to avoid the grid dispersion of more than a few percent (Alford et al., 1974). For the crustal structure and frequency of interest in this study, we used a grid size of $0.025 \mathrm{~km}$. It is also important to make sure that the difference between the exact and the numerical solutions of finite-difference system of equations is bounded with increasing number of grid points for a fixed time interval (Mitchell, 1969). To achieve this, we used a time interval of $0.004 \mathrm{sec}$ using the inequality $\Delta t$ $\leqq h /\left(\alpha^{2}+\beta^{2}\right)^{1 / 2}$ (Alterman and Lowenthal, 1970) where $\alpha$ and $\beta$ correspond to the $P$ - and $S$-wave velocities, and $h$ to the grid size. We also selected a 50-sec time window to be computed. Figure 11 shows three such profiles for three different cases. These seismograms were com-

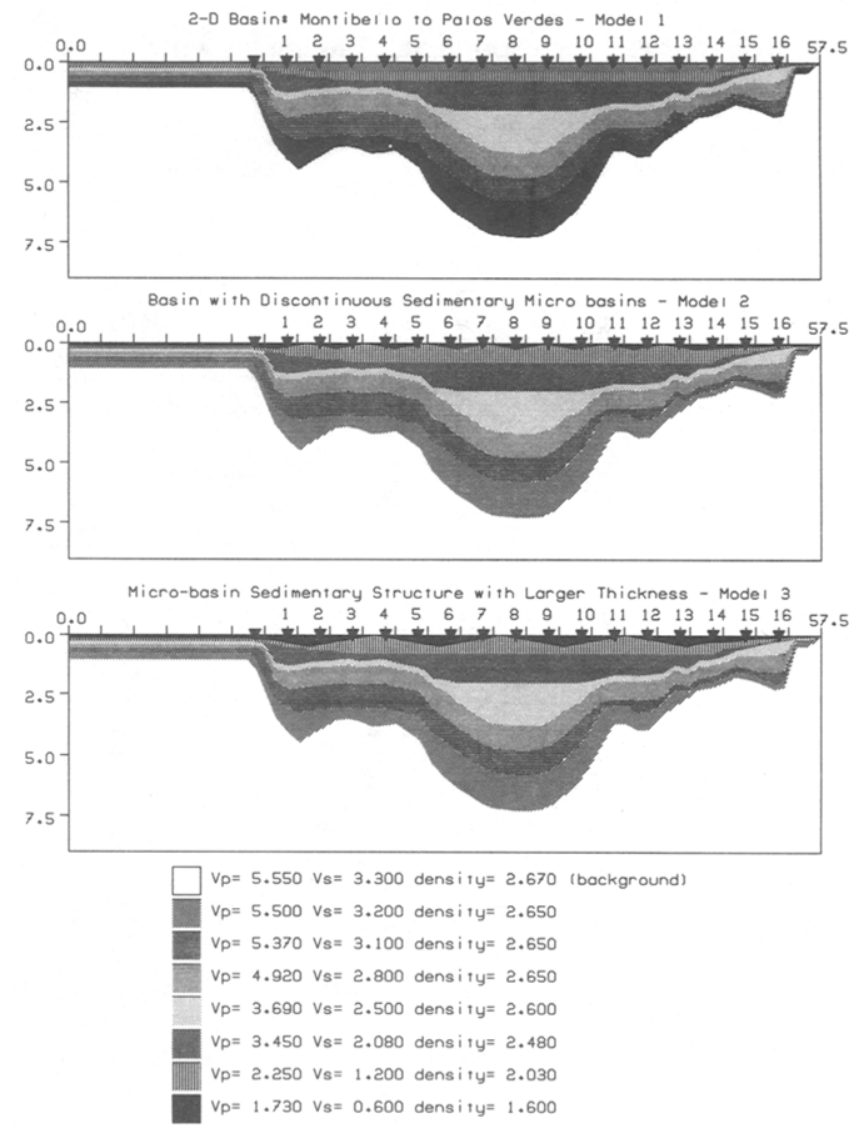

Figure 10. Schematic diagrams of two-dimensional structure used to simulate the finite-difference seismograms. (a) This profile has a continuous low-velocity sedimentary layer at the surface with a $P$ velocity of $1.73 \mathrm{~km} / \mathrm{sec}$ and an $S$ velocity of $0.6 \mathrm{~km} / \mathrm{sec}$, (b) the low-velocity layer is introduced as discontinuous microbasin structure, and (c) the size of microbasin is increased. 
puted using the point-source approximation. Model 1 is the case in which the uppermost layer is a uniform sedimentary layer having a $P$-wave velocity of $1.73 \mathrm{~km} / \mathrm{sec}$ and a $S$-wave velocity of $0.6 \mathrm{~km} / \mathrm{sec}$. Model 2 is the case in which the pockets of low-velocity materials rather than the uniform layer are included in the uppermost crustal layer. Each of these pockets has the same sedimentary materials having a $P$-wave velocity of $1.73 \mathrm{~km} /$ sec and a $S$-wave velocity of $0.6 \mathrm{~km} / \mathrm{sec}$. Model 3 is another case similar to model 2 except that the size of the sedimentary pockets are larger. Although the peak amplitudes of the seismograms at a receiver do not exhibit much variation from model to model, the seismograms for models 1 and 2 do show sustained high-frequency coda energy following the onset of seismic wave field. The coda waves start to develop more strongly at the receivers when the impedance contrast across the pockets of low-velocity sedimentary materials is made larger. To simulate the actual interaction of seismic waves with the subsurface geological units of the basin, the distribution of the shear-wave velocities within the sedimentary pockets should be consistent with the subsurface geology. In the absence of detailed information for all parts of the alluvial basin, the age and the textural character of surficial geological materials available in parts of the Los Angeles basin is useful for constructing such a model. Fumal and Tinsley (1985) determined shearwave velocities in the mappable late quaternary sedimentary units in Los Angeles basin and observed that the shear-wave velocities vary between 0.2 and 0.365 $\mathrm{km} / \mathrm{sec}$ and between 0.305 and $0.65 \mathrm{~km} / \mathrm{sec}$ for fine to very coarse holocene and late and middle pleistocene geological units, respectively. Tinsley and Fumal (1985) used these velocities to construct a generalized shearwave velocity map for the Los Angeles basin (see their Fig. 44). They assumed that these velocities would possibly extend to a depth of significant shaking response during a possible large earthquake. Thus, the depth dis-

\section{RESPONSE OF THREE IRREGULAR BASIN STRUCTURES}

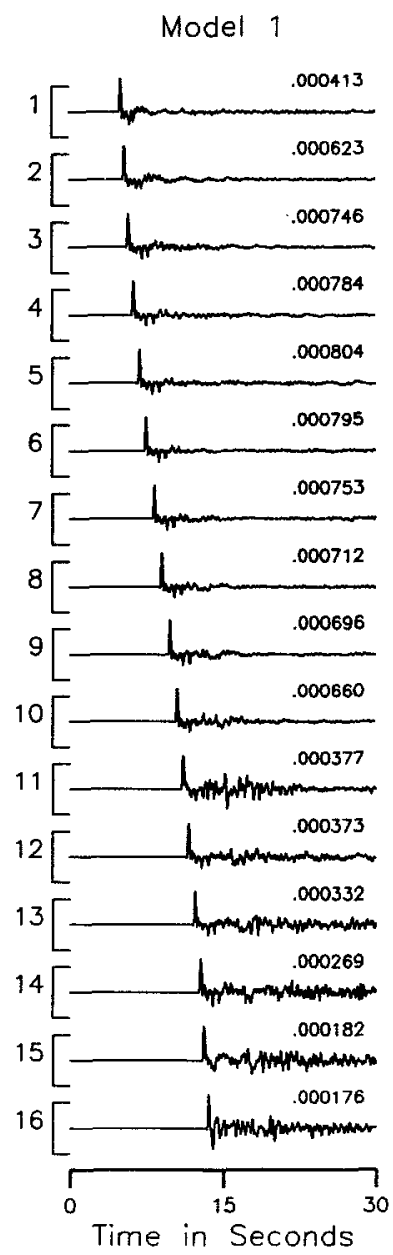

Model 2

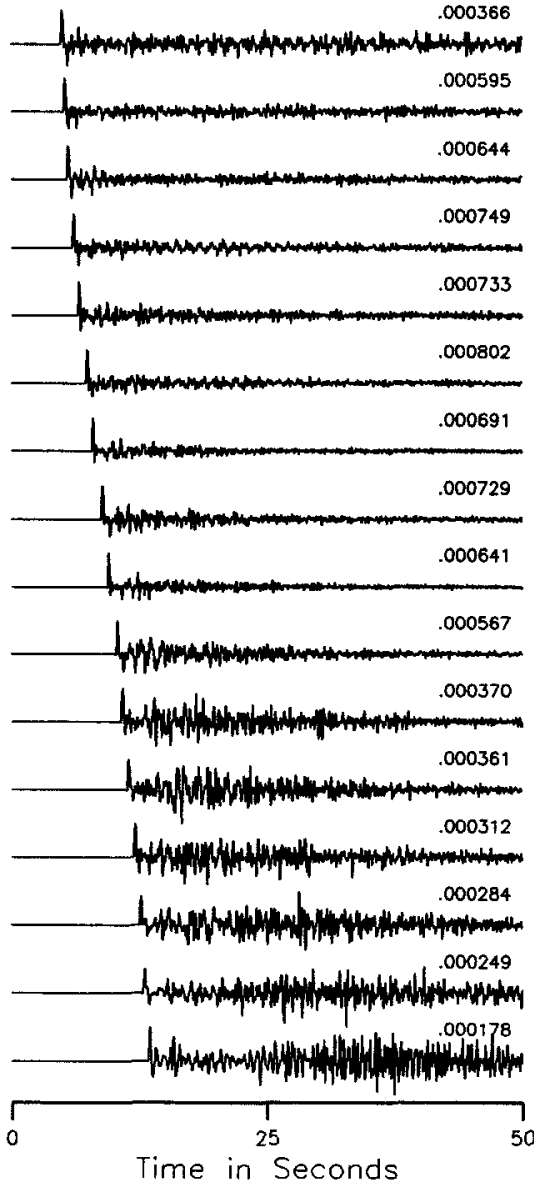

Model 3

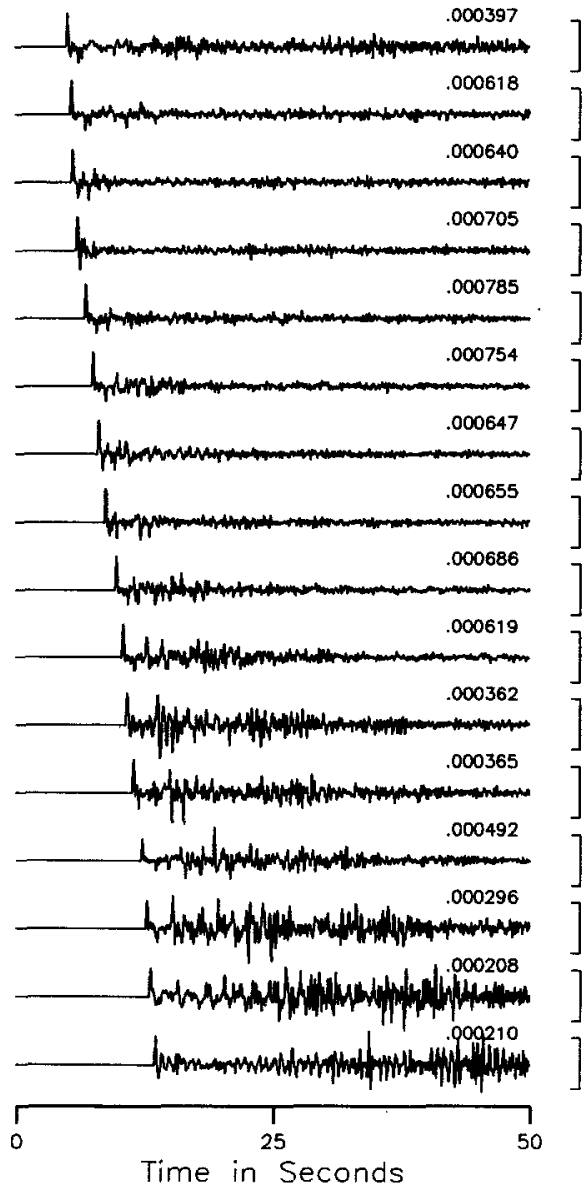

Figure 11. Profiles of finite-difference point-source $S H$ seismograms computed using the crustal models shown in Figure 10. The variation in the level of ground motion and duration of the signals appear to increase with the addition of microbasins into the model. 
tribution of these units is quite ad hoc. Therefore, we have chosen the depth distribution of the low-velocity sedimentary pockets also in an ad hoc manner and assigned a shear-wave velocity of $0.6 \mathrm{~km} / \mathrm{sec}$ within these pockets to investigate the possible effect on the wave propagation. The effect of attenuation is likely to be significant if the shear-wave velocity is small and sizes of the pockets are sufficiently large. Also, the amplification is expected to be large for a large-impedance contrast. Thus, the damping effect of seismic waves within the lowvelocity pockets is likely to be reduced by the amplification caused by the impedance contrast. A relatively large shear-wave velocity within these pockets may adequately represent the total effect of the two phenomena.

We convolved the responses shown in Figure 11 with the tangential component wave field recorded at the CIT station from the MAST explosion, as was done earlier in Figure 5. The result is shown in Figure 12. These seismograms are simulated semi-empirically using a method in which the interfacing of regional wave field with the RSTF response is quite approximate. In reality, it is quite possible that separate RSTF may more accurately represent the effect of various wave types. The uppermost seismogram is the wave field recorded at the CIT station. The profiles of seismograms shown in Figure 12a cor- respond to those simulated using the response of model 1 , and those of Figures $12 \mathrm{~b}$ and $12 \mathrm{c}$ correspond to the simulated seismograms using the crustal response of model 2 and model 3, respectively, using RSTF. The surface waves are sustained for almost $100 \mathrm{sec}$ in all these seismograms, suggesting that the laterally varying structure together with pockets of discontinuous low-velocity materials exposed at the surface of the basin is probably responsible for the observed duration of the long-period surface wave. We have not included $Q$ (anelasticity) in this study. The effect of $Q$ is to attenuate the high-frequency signals more rapidly compared to the low-frequency signals. Thus, it is possible that inclusion of $Q$ would perhaps reduce the amplitudes of waves prior to the surface waves significantly. It has also been shown that an anelastic medium reduces the amplitude and increases the duration of Love waves as it propagated in a basin (Frankel and Vidale, 1992). However, it is difficult to quantify this effect without actually performing the numerical experiments. In reality, the effect of the low-velocity pockets of sediments will be quite complex considering that the response of the basin is truly a threedimensional phenomenon (Frankel and Vidale, 1992).

Figure 13 shows the variation of the temporal energy at various locations in the basin for the three crustal models

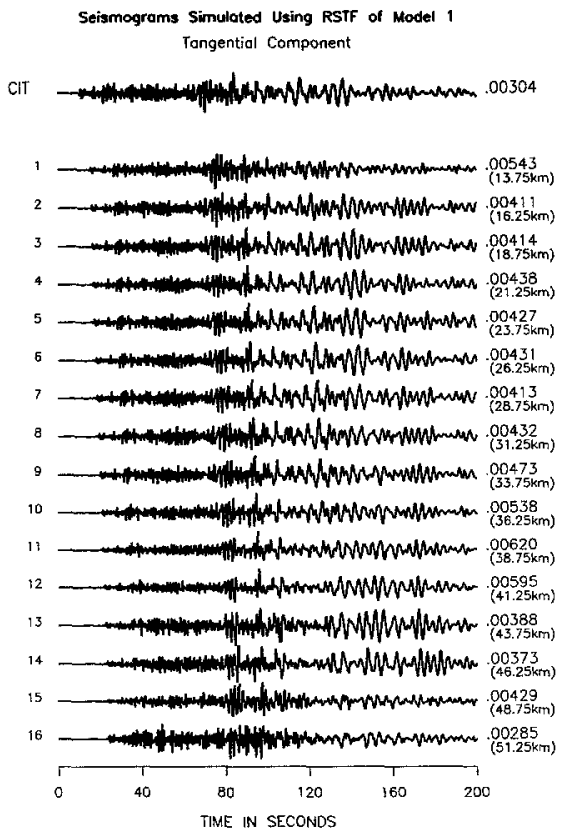

(a)

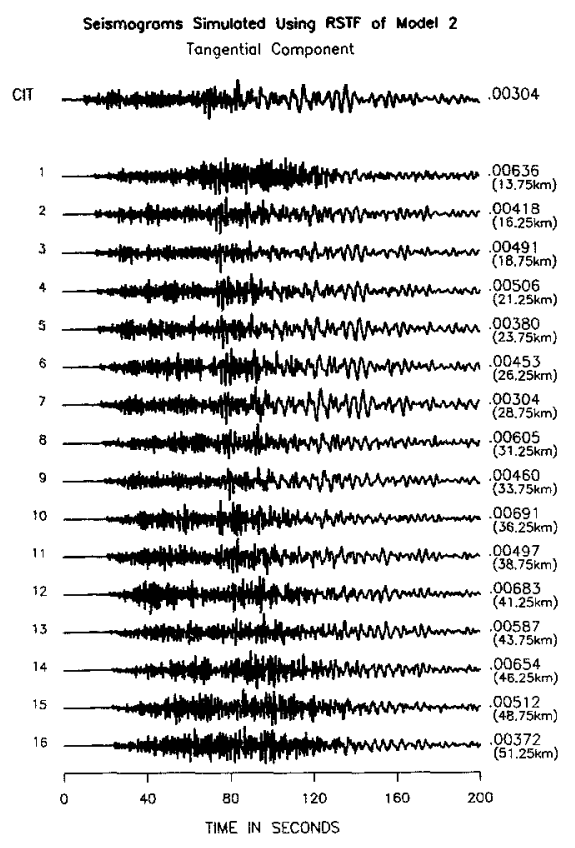

(b)

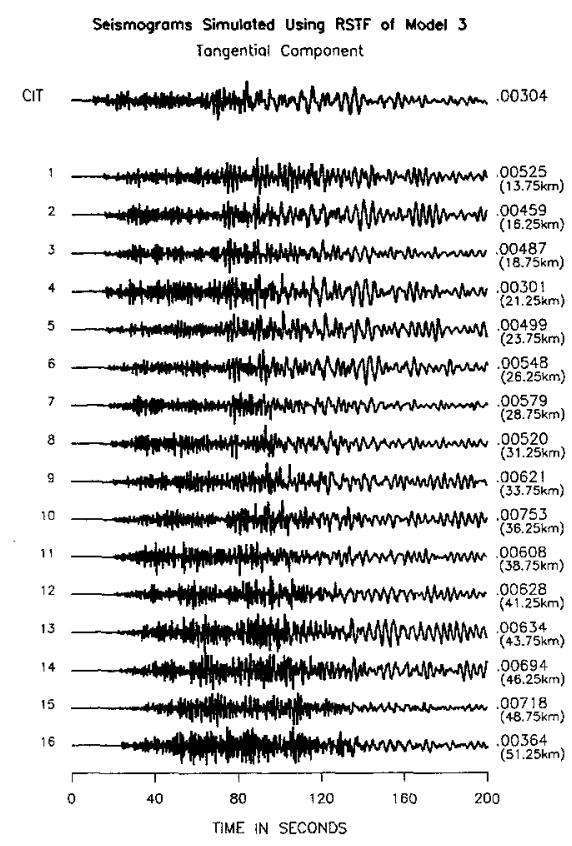

(c)

Figure 12. (a) Profiles of seismograms computed by convolving the recorded seismograms at the CIT station from the MAST explosion with the responses shown for model 1 in Figure 11. The amplitude is normalized as discussed in the text. (b) Profiles of seismograms computed by convolving the recorded seismograms at the CIT station from the MAST explosion with the responses shown for model 2 in Figure 11. The amplitude is normalized as discussed in the text. (c) Profiles of seismograms computed by convolving the recorded seismograms at the CIT station from the MAST explosion with the responses shown for model 3 in Figure 11. The amplitude is normalized as discussed in the text. 
cited above. The variation observed in the left panel of Figure 13 is solely due to the trapping of seismic energy caused by the variation in the basement structure. The character of the temporal energy changes significantly with the introduction of the discontinuous pockets of lowvelocity materials in the upper medium. As discussed previously, these discontinuous pockets delay the seismic waves each time they travel through them. Consequently, the energy appears at a later time on these seismograms, which causes a site-dependent variation in the temporal energy curve. This is similar to the situation observed in the other two panels of Figure 13. In the right panel of Figure 13, the variation is predicted to be about a factor of 9 . In this investigation, we are attempting to demonstrate that a viable means to explain the energy variation observed from site to site. To map this variation exactly, it will be needed to include the actual in situ sedimentary microbasin structures. We could not do this because a detailed mapping of these structures is not yet available. So at this stage, we have tested our proposed method using some canonical models of the sedimentary microbasins.

\section{Conclusions}

We have made several observations on the wavefield amplification in the laterally varying Los Angeles basin structure by analyzing the data from NTS explosions recorded at the various sites of the basin. In the following, we summarize our observations and discuss how we have explained some of the phenomena based on numerical experiments.

The NTS seismograms recorded at several stations of Los Angeles basin from individual explosions exhibit characteristic variation of waveform. This suggests that the response of the basin to the incoming wave field is laterally varying. We also investigated the temporal energy functions computed using the time-domain seismograms and found the level of temporal energy to vary from one site to another. Because the stations are located

\section{Theoretical Temporal Energy Variation at LA Basin Stations}

Model 1

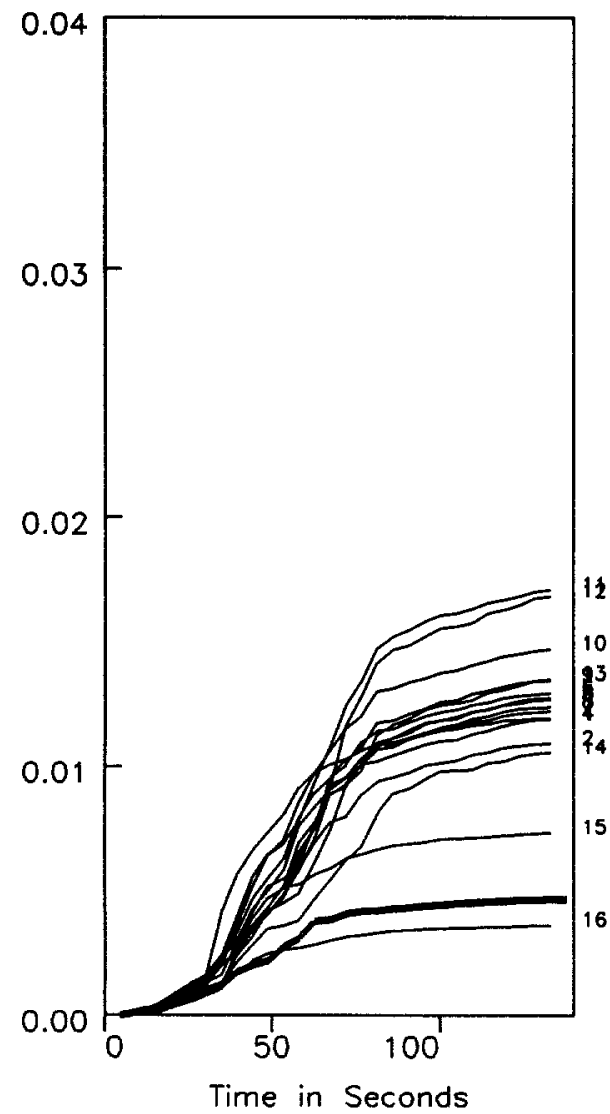

Model 2

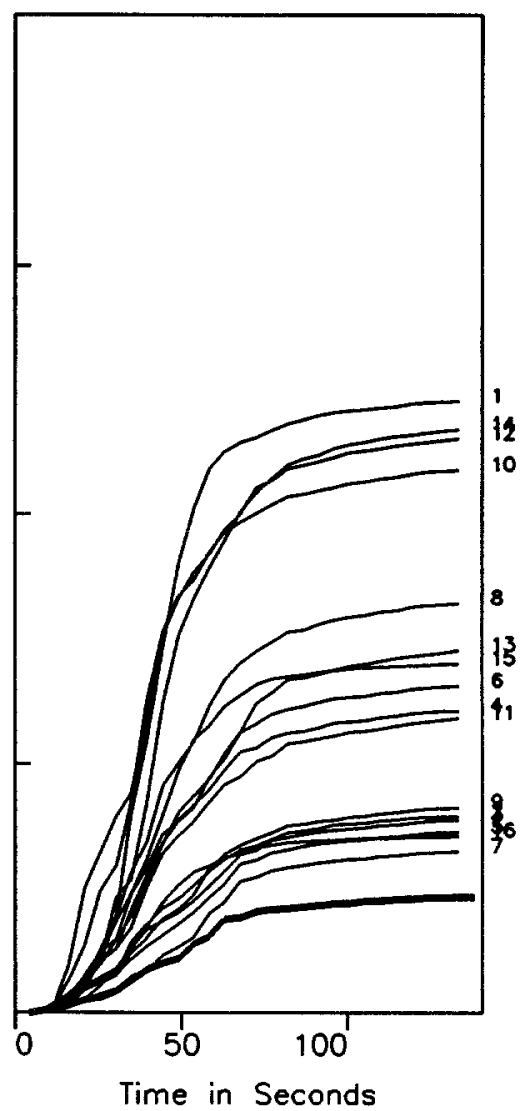

Model 3

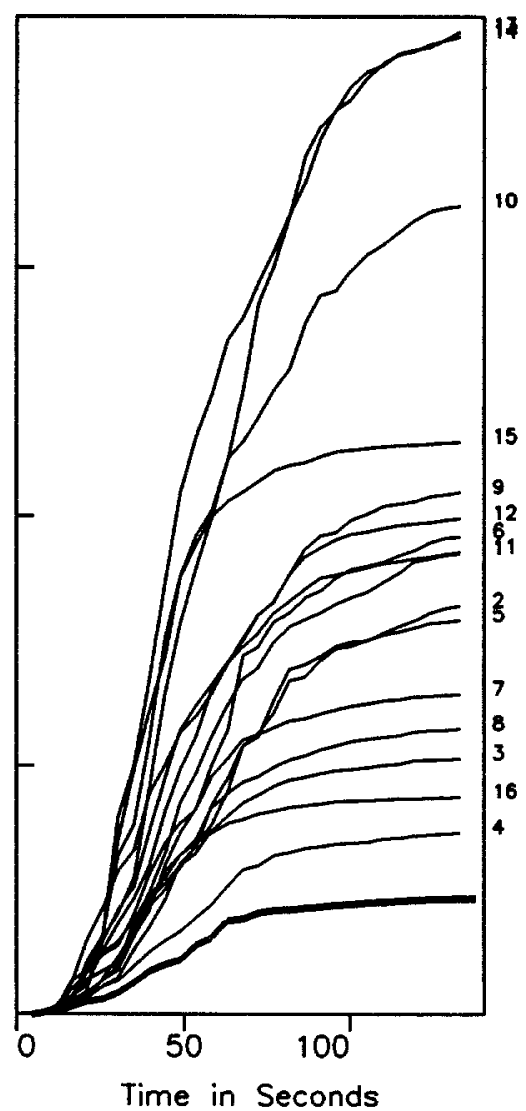

Figure 13. The variation temporal energy as function of sites from the corresponding seismograms shown in Figure 12. These temporal energy curves are obtained in the same manner as those in Figure 6. 
at nearly at the same azimuth, the variation in the temporal energy suggests that the basin is trapping wave field within its own local wave guide.

The seismograms recorded at various stations of downtown Los Angeles around the civic center recorded significantly large long-period surface waves (of approximately a $3-\mathrm{sec}$ period). We found that these longperiod surface waves did not develop at the neighboring hard-rock site at the CIT station. Thus, we concluded that these long-period waves at downtown Los Angeles were caused by a local wave-guide phenomenon. Stratigraphically, there exists a thin sedimentary structure dipping gently toward downtown from the CIT station. Numerical analysis using generalized ray theory indicated that the generation of these long-period waves is probably due to trapping of wave field within this gently dipping sedimentary structure near the downtown stations.

The surface waves recorded at Long Beach from the MAST explosion show long durations of up to $100 \mathrm{sec}$. The wave field recorded at the CIT station from the same explosion does not have these waves over such a long duration. Based on the simulated seismograms obtained by convolving the finite-difference $\mathrm{SH}$ response of the basin for a two-dimensional geological cross section with the CIT station wave field, we were able to generate the surface waves with a duration comparable to that observed at the Long Beach sites. We found that the discontinuous pockets of low-velocity sedimentary structure that are randomly distributed at various parts of the basin could be acting as delay operators to the incoming wave field causing the surface waves to extend over such a long duration. Our study suggests that the size of the individual low-velocity sedimentary structures can be quite critical in defining duration of recorded ground motions. Therefore, it is important to map the shear-wave velocity and the depth distribution of individual subsurface geological units in great detail so that these effects can be represented in the numerical response of a given site.

\section{Acknowledgments}

Special thanks are due to A. M. Rogers of the U. S. Geological Survey who made the digital data available to us. The comments and critical reviews provided by Paul Spudich and by an anonymous reviewer helped to improve this article significantly. The comments from John E. Vidale are highly appreciated. This project was funded by the National Earthquake Hazard Reduction Program (NEHRP) sponsored by the U.S. Geological Survey through the contract 14-08-0001G1684 at Woodward-Clyde Consultants and through the contract 143492-G-2180 at the California Institute of Technology.

\section{References}

Alford, R. M., K. R. Kelly, and D. M. Boore (1974). Accuracy of finite-difference modeling of the acoustic wave equation, Geophysics 39, 834-842.
Alterman, Z. and F. C. Karal (1968). Propagations of elastic waves in layered media by finite-difference methods, Bull. Seism. Soc. Am. 58, 367-398.

Alterman, Z. and D. Lowenthal (1970). Seismic waves in a quarter and three-quarter plane, Geophys. J. R. Astr. Soc. 20, 101-126.

Borcherdt, R. D., W. B. Joyner, R. E. Warrick, and J. F. Gibbs (1975). Response of local geologic units to ground shaking, in Studies for Seismic Zonation of San Francisco Bay Region, U.S. Geol. Surv. Profess. Pap. 94IA, 52-67.

Davis, T. L., J. Namson, and R. B. Yerkes (1989). A cross-section of the Los Angeles area: seismically active fold and thrust belt, the 1987 Whittier Narrows earthquake, and earthquake hazard, J. Geophys. Res., 94, 9644-9664.

Dreger, D. S. and D. V. Helmberger (1990) Broadband modeling of local earthquakes, Bull. Seism. Soc. Am. 80, 1162-1179.

Fumal, T. E. and J. C. Tinsley (1985). Mapping shear-wave velocities of near-surface geological materials, in Evaluating Earthquake Hazards in the Los Angeles Region-An Earth Science Perspective, U.S. Geol. Surv. Profess. Pap. 1360, 127-150.

Frankel, A. and J. Vidale (1992). A three-dimensional simulation of seismic waves in the Santa Clara Valley, California from a Loma Prieta aftershock, Bull. Seism. Soc. Am. 82, 2045-2074.

Helmberger, D. V., G. Engen, and S. Grand (1985). Notes on wave propagation in laterally varying structure, J. Geophys. 58, 8291.

Idriss, I. M. and H. B. Seed (1968). Analysis of ground motions during the 1957 San Francisco earthquake, Bull. Seism. Soc. Am. 58, 2013-2032.

Kawase, H. (1988). Time-domain response of a semicircular canyon for incident $S V, P$, and Rayleigh waves calculated by discrete wavenumber boundary element method, Bull. Seism. Soc. Am. 78, 1415-1437.

Kawase, H. and K. Aki (1989). A study on the response of a soft basin for incident $S, P$, and Rayleigh waves with special reference to the long duration observed in Mexico city, Bull. Seism. Soc. Am. 79, 1361-1382.

Kawase, H. and K. Aki (1990). Topography effect at the critical $S V$ wave incidence: possible explanation of damage pattern by Whittier Narrows, California, Earthquake of 1 October 1987, Bull. Seism. Soc. Am. 80, 1-23.

Mitchell, A. R. (1969). Computational Methods in Partial Differential Equations, John Wiley \& Sons, New York.

Rogers, A. M., P. A. Covington, R. B. Park, R. D. Borcherdt, and D. M. Perkins (1980). Nuclear Events Time Histories and Computed Site Functions for Locations in Los Angeles Region, U.S. Geol. Surv. Open-File Rept. 80-1173, 207 pp.

Rogers, A. M., J. C. Tinsley, W. W. Hays, and K. W. King (1979). Evaluation of the relation between near-surface geological units and ground response in the vicinity of Long Beach, California, Bull. Seism. Soc. Am. 69, 1603-1622.

Saikia, C. K. and L. J. Burdick (1991). Fine structure for Pnl for explosions, J. Geophys. Res. 96, 14,383-14,401.

Seed, H. B. and I. M. Idriss (1968). Seismic response of horizontal soil layers, J. Soil Mechan. Found. Div. ASCE, 94, SM4, 10031031.

Seed, H. B., R. V. Whitman, H. Dezfulian, R. Dobry, and I. M. Idriss (1972). Soil conditions and building damage in the 1967 Caracas earthquake, J. Am. Soc. Civil. Eng. SM Div. 98, 787806.

Tinsley, J. C. and T. E. Fumal (1985). Mapping quaternary sedimentary deposits for areal variations in shaking response, in Evaluating Earthquake Hazards in the Los Angeles Region-An Earth Science Perspective, U.S. Geol. Surv. Profess. Pap. 1360, 101-126.

Vidale, J. E. and D. V. Helmberger (1988). Elastic finite-difference 
modeling of the 1971 San Fernando, California, earthquake, Bull. Seism. Soc. Am. 78, 122-141.

Vidale, J. E., D. V. Helmberger, and R. W. Clayton (1985). Finitedifference seismograms for $S H$ waves, Bull. Seism. Soc. Am. 75, 1765-1782.

Wright, T. L. (1987). Structural geology and tectonic evolution of the Los Angeles basin, Presented at the Annual Meeting of the AAPG.

Yerkes, R. F., T. H. McCulloh, J. E. Schoellhamer, and J, G. Vedder (1965). Geology of Los Angeles Basin, California, U.S. Geol. Surv. Profess. Pap. 420-A, 57 pp.
Woodward-Clyde Consultants

566 El Dorado St.

Pasadena, California 91101

$$
\text { (C.K.S.) }
$$

Seismological Laboratory

California Institute of Technology

Pasadena, California 91125.

(D.S.D. and D.V.H.)

Manuscript received 28 May 1992. 